\title{
Brain Serotonin Transporter Occupancy by Oral Sibutramine Dosed to Steady State: A PET Study Using " C-DASB in Healthy Humans
}

\author{
Peter S Talbot*,', Stefan Bradley², Cyril P Clarke², Kola O Babalola ${ }^{3}$, Andrew W Philipp ${ }^{2}$, Gavin Brown ${ }^{4}$, \\ Adam W McMahon ${ }^{4}$ and Julian C Matthews ${ }^{4}$ \\ 'Wolfson Molecular Imaging Centre, The University of Manchester, Manchester, UK; ${ }^{2}$ Icon Development Solutions, Manchester, UK; ${ }^{3}$ Manchester \\ Academic Health Science Centre, Stopford Building, The University of Manchester, Manchester, UK; ${ }^{4}$ Manchester Academic Health Science \\ Centre, Wolfson Molecular Imaging Centre, The University of Manchester, Manchester, UK
}

\begin{abstract}
Sibutramine is a centrally acting monoamine reuptake inhibitor prescribed as an appetite suppressant in the management of obesity. Its effects are mostly attributable to serotonin and norepinephrine transporter (SERT and NET, respectively) inhibition by its potent metabolites mono-desmethylsibutramine (MI) and di-desmethylsibutramine (M2). However, there is a paucity of in vivo data in humans about mechanisms underlying both clinical efficacy and the dose-independent non-response observed in a minority of patients. Twelve healthy male patients (mean age 4 I years) completed a double-blind, placebo-controlled, within-subject crossover investigation of brain SERT occupancy by sibutramine $15 \mathrm{mg}$ daily at steady state. Correlations were measured between occupancy and (i) plasma concentrations of sibutramine, MI and M2; (ii) appetite suppression. " 'C-DASB PET scans were performed on the HRRT camera. Binding potentials $\left(\mathrm{BP}_{\mathrm{ND}}\right.$ ) were calculated by the Logan reference tissue (cerebellum) method. SERT occupancy was modest (mean $30 \pm 10 \%$ ), was similar across brain regions, but varied widely across subjects (15-46\%). Occupancy was correlated positively $(p=0.09)$ with $\mathrm{M} 2$ concentration, but not with sibutramine or MI. No significant appetite suppression was seen at $<25 \%$ occupancy and greatest suppression was associated with highest occupancy (25-46\%). However, several subjects with occupancy (36-39\%) in the higher range had no appetite suppression. SERT occupancy by clinical doses of sibutramine is of modest magnitude and may be mediated predominantly by $\mathrm{M} 2$ in humans. 5-HT reuptake inhibition may be necessary but is not sufficient for sibutramine's efficacy in humans, supporting preclinical data suggesting that the hypophagic effect requires the co-inhibition of both SERT and NET.

Neuropsychopharmacology (2010) 35, 74I-75I; do::I0.1038/npp.2009. I82; published online 4 November 2009
\end{abstract}

Keywords: sibutramine; appetite suppression; serotonin transporter (SERT); positron emission tomography $(\mathrm{PET}) ;{ }^{1} \mathrm{C}$-DASB; High Resolution Research Tomograph (HRRT)

\section{INTRODUCTION}

Sibutramine hydrochloride is an orally administered centrally acting monoamine reuptake inhibitor. It is licensed as an anti-obesity agent because of its appetite suppressant and thermogenic effects, and promotes modest weight loss when given along with recommendations for diet (Arterburn et al, 2004; Hainer et al, 2006; Li et al, 2005; Padwal et al, 2003). Its efficacy would appear to be due to the co-inhibition of central serotonin (5-HT) and norepinephrine (NE) reuptake transporters (SERT and NET,

\footnotetext{
*Correspondence: Dr PS Talbot, Wolfson Molecular Imaging Centre, The University of Manchester, 27 Palatine Road, Withington, Manchester, Greater Manchester, M20 3LJ, UK,

Tel: + 440 |6| 2750015 , Fax: + 440 161 2750003 ,

E-mail: peter.talbot@manchester.ac.uk

Received 30 July 2009; revised 2 October 2009; accepted 6 October 2009
}

respectively) resulting in increased extracellular 5-HT and NE (Jackson et al, 1997b), subsequent activation of a variety of 5-HT and NE receptor systems (Grignaschi et al, 1999; Jackson et al, 1997a; Liu et al, 2002), and increased sympathetic activity to brown adipose tissue (Liu et al, 1996, 2002). Sibutramine itself is a relatively weak NET inhibitor, and a very weak SERT inhibitor, in vitro (Buckett et al, 1988). However, its secondary ( $N$-mono-desmethylsibutramine; BTS 54354; Metabolite 1 ) and primary ( $N$-didesmethylsibutramine; BTS 54505; Metabolite 2) amine metabolites are both potent SERT and NET inhibitors in vitro, having approximately 100 -fold higher affinity than parent sibutramine (Cheetham et al, 1993; Heal et al, 1998; Luscombe et al, 1989). The pharmacological effects of sibutramine in vivo are mostly attributable to Metabolites 1 and 2 (M1 and M2).

United Kingdom prescription guidelines recommend a starting dose of $10 \mathrm{mg}$ daily in a single dose (usually 
morning), increasing to a maximum of $15 \mathrm{mg}$ daily after 4 weeks (BNF, 2009). Higher doses are associated with a high incidence of cardiovascular pressor effects and other adverse events (King and Devaney, 1988; Weintraub et al, 1991). Following oral administration, the time to maximum plasma concentration $\left(t_{\max }\right)$ of the active metabolites is $2.5-3.6 \mathrm{~h}$, and is not significantly altered by repeated administration. The elimination half-life $\left(t_{\frac{1}{2}}\right)$ from plasma of the active metabolites is $14-19 \mathrm{~h}$. With repeated administration, sibutramine $15 \mathrm{mg}$ daily reaches steady state in plasma by approximately 4 days (Garratt et al, 1995; McNeely and Goa, 1998).

In clinical trials of up to 6 months' duration, a minority of patients (approximately 15\%) experience no weight reduction or even a paradoxical increase. This lack of efficacy is not clearly related to the dose of sibutramine received (McNeely and Goa, 1998), and possible mechanisms underlying non-response are poorly understood. In a study of healthy male volunteers administered sibutramine 5, 10 or $20 \mathrm{mg}$ once daily for 14 days, the doses below $20 \mathrm{mg}$ did not reliably inhibit ${ }^{3} \mathrm{H}-5-\mathrm{HT}$ uptake in an in vitro human platelet SERT preparation (Luscombe et al, 1990). Even the $20 \mathrm{mg}$ dose could not be distinguished from placebo, although it showed $20-30 \%$ inhibition. Taken together these data suggest that SERT inhibition at currently recommended clinical doses (10-15 mg daily) may be very low. This suspicion was supported by recent rodent studies showing negligible $(\sim 3 \%)$ SERT occupancy at the minimal effective dose of sibutramine for significant food intake reduction $(1 \mathrm{mg} / \mathrm{kg})$, and low occupancy $(\sim 15-20 \%)$ at doses up to 10-fold higher (Thomas et al, 2009). However, to the best of our knowledge, there are no studies of central SERT inhibition in humans at any dose, and an overall paucity of in vivo data in humans about mechanisms of clinical efficacy and non-response.

The PET radiotracer ${ }^{11} \mathrm{C}-\mathrm{DASB}$ (Wilson et al, 2000) has high affinity and selectivity for the SERT, shows significant advantages over earlier tracers used for SERT quantitation, has excellent test-retest reliability in brain regions with high SERT density, and is widely used for the measurement of SERT occupancy by drugs using established analytical methods (Frankle et al, 2004, 2006; Meyer et al, 2004).

The primary aim of this study was therefore to measure, for the first time, the central SERT occupancy associated with common clinical use of sibutramine in humans, that is, $15 \mathrm{mg} /$ day dosed to steady state, using ${ }^{11} \mathrm{C}-\mathrm{DASB} \mathrm{PET}$. Exploratory secondary analyses investigated correlations between SERT occupancy and pharmacokinetic (PK) and pharmacodynamic (PD) measures: (i) between SERT occupancy and plasma concentrations of parent sibutramine, M1 and M2; and (ii) between SERT occupancy and the magnitude of appetite suppression.

\section{MATERIALS AND METHODS}

\section{Human Subjects}

Healthy males in the age range of 30-55 years and with body mass index (BMI) in the normal range $\left(18-24.9 \mathrm{~kg} / \mathrm{m}^{2}\right)$ were recruited from the Icon Development Solutions volunteer panel. Subjects were required to be in good health on the basis of medical history, physical examination, vital signs,
EKG, and laboratory investigations (including screen for drugs of abuse). Exclusion criteria included: current diagnosis of any Axis I (DSM-IV) disorder; a past history of drug or alcohol dependence; drug or alcohol misuse within the preceding 6 months; any use of MDMA ('Ecstasy'), cigarettes or nicotine preparations in the preceding 3 months; prescribed medications in the preceding 3 weeks (6 weeks for fluoxetine); over-the-counter preparations (except paracetamol (acetaminophen) or multivitamins) in the preceding 7 days; a history of neurological disorder including epilepsy, stroke, migraine, or a history of significant head trauma resulting in loss of consciousness for greater than $10 \mathrm{~min}$; contraindication for sibutramine; subjects evaluated as dieting (score $>11$ ) on the dietary restraint (F1) subscale of the Three-factor Eating Questionnaire (Stunkard and Messick, 1985).

The study was approved by the Manchester Independent Research Ethics Committee and by the United Kingdom Administration of Radioactive Substances Advisory Committee. All subjects provided written informed consent after receiving written and verbal explanations of the study.

\section{Study Design}

Except for the PET and MRI scans, screening and study procedures took place at the ICON Clinical Unit. Following inclusion, all subjects participated in two study periods, each lasting 5 days, in a double-blind, placebo-controlled, balanced-order, within-subject crossover design. In one period (sibutramine condition) subjects received once daily (08:30, following overnight fast) $15 \mathrm{mg}$ doses of racemic sibutramine hydrochloride for 5 days, in a controlled residential setting with standardized diet throughout. On day 4, starting $240 \mathrm{~min}$ post-dose, subjects were served a standardized test meal to measure food intake in grams (PD effect). On day 5, starting $240 \mathrm{~min}$ post-dose, subjects had a brain PET scan with ${ }^{11} \mathrm{C}$-DASB to quantify SERT availability. Fifteen minutes before the test meal and the PET scan, venous blood $(7.5 \mathrm{ml})$ was sampled for PK measurements: plasma concentrations of sibutramine, M1, and M2. Based on the literature, we expected parent sibutramine and metabolites to reach plasma steady state by day 4 , so that the PD (day 4) and PET (day 5) data would be acquired under similar PK conditions. The time of $240 \mathrm{~min}$ post-dose was chosen to be in the early part of the slow washout phase following peak plasma concentrations of M1 and M2. After the PET scan on day 5 the subject's participation in that study period came to an end. However, in the event that we were unable to acquire the PET scan on day 5 because of unforeseen technical problems, we retained the ability to keep the subject in the study for an extra day to acquire the scan.

The other study period (placebo condition) was identical except that subjects received doses of placebo rather than sibutramine (formulated in capsules of identical appearance). The sequence in which subjects participated in the two study periods (sibutramine followed by placebo, or vice versa) was a balanced block-randomization with six subjects per treatment sequence. The study periods were 2 weeks apart, resulting in a drug-free washout of 13-15 days between PET scans for those subjects who received sibutramine in the first study period. A post-study medical 
evaluation was performed within 7 days of completion of the second period. Subjects who withdrew, or were withdrawn, from the study before completion were replaced until 12 subjects completed the study (subject to a maximum recruitment of 16 subjects).

\section{Test Meal Procedure}

On day 4 of both study periods, a standardized test meal (Hill et al, 1995) was served 240 min post-dose consisting of pasta and a tomato and basil sauce well mixed to ensure the pasta was evenly covered with sauce. The energy density of the test meal was $4.71 \mathrm{~kJ} / \mathrm{g}$. Drinking water was provided. Subjects were instructed to eat as much food as they wanted and to indicate when they had finished by pressing a button fixed to the table. This sounded a bell in an adjacent room from where the test meal was being monitored. The remaining food was removed from the room.

The test meal was served on a universal eating monitor (UEM) apparatus (Kissileff et al, 1980) in an individual testing room devoid of distractions. The UEM apparatus consisted of a custom built table with a digital balance (Sartorius CP 4201) concealed beneath such that the top pan of the balance protruded slightly through a hole cut into the table top. The top pan of the balance was covered with a plastic place mat, which was fixed to the table so that no part of the balance was visible. The weight of food remaining was recorded every $3 \mathrm{~s}$ throughout the test meal and was communicated via a serial cable to a computer in an adjacent room. The subject was observed via a hidden closed-circuit television camera so that any artifacts caused by the subject moving the bowl or stirring the food could be removed from the resultant data set. Before the test meal, subjects fasted (water permitted) following a standardized breakfast.

\section{Pharmacodynamic Outcome}

The PD effect of sibutramine was measured as the percent difference in total test meal food intake (grams) between the placebo and sibutramine conditions. The test-retest variability (coefficient of variation (\%CV)) and reliability (intraclass correlation (ICC)) of the test meal were previously established in a separate cohort of 12 placebodosed, normal-weight males as $14.2 \pm 9.5 \%$ and 0.84 , respectively (ICON, unpublished data). Thus, in this study, a clinically significant response to sibutramine was defined as a reduction in food intake of at least $14.2 \%$ in the sibutramine condition compared with the placebo condition.

\section{Pharmacokinetic Measurement}

Venous blood samples on days 4 and 5 were collected in chilled lithium heparin tubes, and immediately centrifuged at $1500 \mathrm{~g}, 4^{\circ} \mathrm{C}$, for $10 \mathrm{~min}$. Plasma was drawn off and stored at $-70^{\circ} \mathrm{C}$. The validated method involved solid phase extraction on Oasis HLB and analysis on a Fortis C18 column by LC-MS/MS. The lower limit of quantitation was $0.5 \mathrm{ng} / \mathrm{ml}$.

\section{Radiochemistry}

The DASB analytical standard and the synthetic precursor desmethyl DASB were obtained from ABX (Radeburg, Germany). Preparation of ${ }^{11} \mathrm{C}$-DASB followed the procedure reported by Huang et al (2004) with some modifications. ${ }^{11}$ C-Methyl iodide (Larsen et al, 1997) was passed under a stream of helium into a reaction vessel containing a solution of desmethyl DASB $(1.0 \mathrm{mg})$ dissolved in $\mathrm{N}, \mathrm{N}$-dimethylformamide $(500 \mu \mathrm{l})$ at $-10^{\circ} \mathrm{C}$. The reaction vessel was sealed and the reaction mixture heated at $85 \mathrm{C}$ for $5 \mathrm{~min}$ with stirring. The reaction mixture was then cooled to $40^{\circ} \mathrm{C}$ and $1.5 \mathrm{ml}$ of $0.1 \mathrm{M}$ ammonium acetate/acetonitrile $(50: 50)$ was added. The resultant solution was injected onto a Phenomenex Prodigy ODS-3 $(250 \times 10 \mathrm{~mm}, 10 \mu \mathrm{m})$ column and eluted with $0.1 \mathrm{M}$ ammonium acetate/acetonitrile $(50: 50)$ at a flow rate of $6 \mathrm{ml} / \mathrm{min}$. The eluent was monitored continuously for both radioactivity and absorbance at $254 \mathrm{~nm}$ and the fraction eluting between 10.5 and $12.5 \mathrm{~min}$, having the same retention time as reference DASB was collected into a flask containing water $(50 \mathrm{ml})$. This solution was passed through a $\mathrm{C}_{18}$ SepPak Plus cartridge (Waters). The SepPak cartridge was rinsed with water $(10 \mathrm{ml})$ and the product eluted with ethanol $(1.0 \mathrm{ml})$. The product in ethanol $(1.0 \mathrm{ml})$ was then diluted with $0.9 \%$ sterile saline $(12 \mathrm{ml})$ and passed through a $0.22 \mathrm{~mm}$ membrane filter (Millex GV, $0.22 \mu \mathrm{m}, 25 \mathrm{~mm}$; Millipore) into a sterile vial. The total radiosynthesis time was $40-45$ min giving $1.2-2.2 \mathrm{GBq}$ of formulated product at the end of the radiosynthesis.

A sample $(50 \mu \mathrm{l})$ of the formulated product was analyzed by HPLC using an ACE C-18HL $3 \mu \mathrm{m}$ column $(150 \times 4.6 \mathrm{~mm})$ eluted at $1.3 \mathrm{ml} / \mathrm{min}$ with $0.1 \mathrm{M}$ ammonium acetate/acetonitrile $(77: 23), \mathrm{pH} 4.7$. The retention time for both reference DASB and the formulated product was $10 \mathrm{~min}$.

\section{PET Protocol}

All scans were performed on a High Resolution Research Tomograph (HRRT; CTI/Siemens Molecular Imaging, TN, USA) (de Jong et al, 2007) at the Wolfson Molecular Imaging Centre. This is a dedicated brain PET scanner comprising eight flat panel detectors, with transaxial and axial FOVs of 31.2 and $25.2 \mathrm{~cm}$, respectively. Depth-ofinteraction information is provided by a dual-layer scintillator (10 $\mathrm{mm}$ LSO, $10 \mathrm{~mm}$ LYSO). Point source resolution varies across the field of view from approximately 2.3 to $3.2 \mathrm{~mm}$ (full-width-at-half-maximum; FWHM) in the transaxial direction and from 2.5 to $3.4 \mathrm{~mm}$ in the axial direction. Attenuation correction is facilitated by a ${ }^{137}$ Cs transmission point source.

${ }^{11} \mathrm{C}$-DASB injection was scheduled for $240 \mathrm{~min}$ post-dose. However, given the practical difficulties in ensuring exact scan start times, and the long washout time of the M1 and M2 metabolites, we felt $a$ priori that a 30 min margin would be acceptable. We therefore aimed for radiotracer injection 240-270 min post-dose, and for each subject's 2 PET scans to start within $30 \mathrm{~min}$ of each other. Where a subject's first scan was unavoidably delayed beyond $270 \mathrm{~min}$, every effort was made to delay the commencement of their second scan by the same duration, so that ${ }^{11} \mathrm{C}$-DASB binding potentials 
across the two conditions would be measured under comparable PK conditions. Fifteen minutes before tracer injection a venous cannula was inserted in an antecubital vein in the non-dominant arm, blood was sampled for PK measurement, and the cannula left in situ for subsequent

${ }^{11} \mathrm{C}$-DASB injection.

Head movement within the HRRT head holder was monitored by the Polaris Vicra infrared motion detection device (Northern Digital, Ontario, Canada), with the reflective tool attached to the vertex of a customized neoprene swim cap. Head movement was reduced by the snug fit of the cap within the head holder and the use of velcro forehead and chin straps. A 6 min transmission scan was performed before injection. ${ }^{11} \mathrm{C}$-DASB was injected by i.v. bolus over $20 \mathrm{~s}$, following which emission data were acquired in list mode for $100 \mathrm{~min}$. The emission data were rebinned into 27 frames of increasing duration (span 9, max. ring difference $=67$ ). Randoms correction was calculated from delayed coincidences using a variance reduction technique (Byars et al, 2005). Scatter correction was calculated via simulation (Watson, 2000).

\section{MRI Acquisition}

A $\mathrm{T}_{1}$-weighted MRI brain scan (to exclude structural abnormality, and for identification of regions of interest (ROI) for PET scan analysis) was acquired on a 1.5 Tesla Phillips ACS MR system using a 3-D magnetization prepared rapid gradient echo sequence (MPRAGE; TR $8.6 \mathrm{~ms}$, TE $4.0 \mathrm{~ms}$, FOV $240 \mathrm{~mm}^{2}$, Matrix $256 \times 256$, reconstructed voxel size $0.94 \times 0.94 \times 1.2,170$ slices) and clinically reported before each subject's first PET scan.

\section{Image Analysis}

PET images were reconstructed to a $256 \times 256 \times 207$ matrix (isotropic voxel size of $1.22 \mathrm{~mm}^{3}$ ) using an iterative algorithm, Ordinary Poisson-Ordered Subset Expectation Maximization (OP-OSEM; 12 iterations, 16 subsets) (Michel et al, 2000; van Velden et al, 2009). Attenuation correction was calculated using a segmented $\mu$-map image (Sibomana et al, 2004).

For each subject, the following SERT-rich ROIs were extracted from their $\mathrm{T}_{1}$-weighted MRI using an automated method (Babalola et al, 2008): putamen (PUT), caudate (CAU), thalamus (THA) and brainstem (BS; comprising SERT-rich midbrain plus pons and medulla). The region of reference (ROR) was manually delineated on the MRI, using Analyze (Mayo Clinic, MN, USA), in posterior cerebellar cortex, excluding vermis and central white matter and keeping at least one FWHM from venous sinuses and occipital cortex (Meyer, 2007). Using reconstructed images over the entire duration of the scan, rigid body registration parameters were determined using Vinci (Max-Planck Institute, Cologne, Germany) and the MMM co-registration module (normalized mutual information metric), and subsequently used to transform the delineated regions to both scans using in-house MATLAB (The MathWorks, Natick, MA, USA) code. Time activity curves were calculated for each region from the 27-frame dynamic scan data using in-house software incorporating Analyze AVW libraries.

\section{Derivation of ${ }^{11} \mathrm{C}$-DASB Regional Binding Potentials}

Regional binding potentials $\left(\mathrm{BP}_{\mathrm{ND}}\right.$; the ratio at equilibrium of specifically bound radioligand to that of nondisplaceable radioligand in tissue) (Innis et al, 2007) were derived using a local MATLAB implementation of the reference tissue Logan method (Logan et al, 1996) and calculated as distribution volume ratio minus one (DVR-1). A pseudoequilibrium of the ROR and ROI is assumed. The use of the Logan method avoids requiring the single tissue compartment assumption across different levels of SERT occupancy (Meyer, 2007). SERT occupancy was defined as percentage of change in $\mathrm{BP}_{\mathrm{ND}}$ between placebo and sibutramine conditions. $\mathrm{BP}_{\mathrm{ND}}$ and occupancy values were finalized before correlations with $\mathrm{PD}$ and $\mathrm{PK}$ parameters were conducted and remained unaltered thereafter.

\section{RESULTS}

\section{Human Subjects}

Fifteen subjects were enrolled, of whom 12 completed the study (mean age 40.5 years, range $30-50$ years; mean BMI $22.9 \mathrm{~kg} \mathrm{~m}^{-2}$, range $\left.20.3-24.7\right)$. Of the additional three subjects, one was withdrawn before the second PET scan because of opiate drugs of abuse on urine screening and non-compliance with caffeine and alcohol restrictions; one withdrew his consent before the first PET scan due to nausea on sibutramine; and one patient had consecutive ${ }^{11} \mathrm{C}$-DASB radiochemistry failures on days 5 and 6 of the first study period.

\section{PET Scans}

Start times. No scans were commenced before the intended 240 min post-dose start time. Median PET scan start time was 247 min (range 241-319) for the placebo condition and 257 min (range 242-343) for the sibutramine condition. For eight subjects, both scans were commenced by $270 \mathrm{~min}$ post-dose. For two additional subjects, both scans were delayed beyond $270 \mathrm{~min}$ (placebo and sibutramine times: 284 and $278 \mathrm{~min} ; 319$ and $343 \mathrm{~min}$, respectively) but remained within \pm 30 min of each other. For the final two subjects, although the placebo scan started by $270 \mathrm{~min}(243$ and $244 \mathrm{~min}$ ) the sibutramine scan was delayed beyond $270 \mathrm{~min}$ (277 and $284 \mathrm{~min}$, respectively). Thus, their scan start times were more than $30 \mathrm{~min}$ apart (34 and $40 \mathrm{~min}$, respectively).

\begin{abstract}
${ }^{11} \mathrm{C}$-DASB injected doses. ${ }^{11} \mathrm{C}$-DASB injected dose $(498.5 \pm 105.8 \mathrm{MBq} \quad(13.5 \pm 2.9 \mathrm{mCi}))$, injected mass $(4.5 \pm 2.6 \mu \mathrm{g})$, specific activity at the time of injection $\left(41.4 \pm 21.6 \mathrm{GBq} \mathrm{mol}^{-1} \quad\left(1119 \pm 583{\left.\left.\mathrm{mCi} \mu \mathrm{mol}^{-1}\right)\right)}^{-1}\right.\right.$ and radiochemical purity $(96.7 \pm 1.1 \%)$ did not differ between placebo and sibutramine conditions (paired $t$-test, twotailed; $p=0.94,0.66,0.96$, and 0.91 , respectively).
\end{abstract}

\section{Binding Potentials and SERT Occupancy}

Values for ${ }^{11} \mathrm{C}$-DASB $\mathrm{BP}_{\mathrm{ND}}$ across regions in the placebo and sibutramine conditions, and corresponding SERT occupancy by sibutramine, are shown in Table 1. Mean SERT occupancy across the four regions was 
$30.0 \pm 10.2 \%$. There was a highly significant degree of variability in occupancy between subjects $(\mathrm{F}[11,36]=6.29$, $p<0.00005)$, ranging from 14.6 to $45.6 \%$. There was also a

Table I Regional "'C-DASB Binding Potentials (BP $\mathrm{ND}$ ) Under Placebo and Sibutramine Conditions, and Corresponding 5-HT Transporter Occupancy Values

\begin{tabular}{lccc}
\hline \multirow{2}{*}{ Region } & \multicolumn{2}{c}{ Binding potential (BP $\left.\mathbf{P D}_{\mathbf{N D}}\right)$} & Occupancy (\%) \\
\cline { 2 - 3 } & Placebo & Sibutramine & \\
\hline THA & $1.25 \pm 0.31$ & $0.93 \pm 0.22$ & $24.6 \pm 8.8$ \\
PUT & $1.22 \pm 0.32$ & $0.86 \pm 0.25$ & $29.0 \pm 9.0$ \\
CAU & $0.82 \pm 0.24$ & $0.55 \pm 0.19$ & $32.8 \pm 13.6$ \\
BS & $0.60 \pm 0.09$ & $0.40 \pm 0.06$ & $33.5 \pm 6.8$ \\
\hline
\end{tabular}

THA, thalamus; PUT, putamen; CAU, caudate nucleus; BS, brainstem.

Values represent group $(n=12)$ mean \pm SD. substantially smaller, but still statistically significant, degree of variability in occupancy between regions $(F[3,33]=6.28$, $p=0.002$ ), once variability across subjects was accounted for, with measured occupancy being smaller in THA. ANOVA confirmed that a greater proportion of the sum of squares variability was across subjects (66\%) than regions $(12 \%)$. Figure 1 (Panels $\mathrm{a}$ and $\mathrm{b}$ only) illustrates the modest attenuation of ${ }^{11} \mathrm{C}$-DASB binding by sibutramine in a representative subject.

\section{Sibutramine Pharmacokinetics}

Plasma concentrations. Parent sibutramine, M1 and M2 concentrations were assayed on day 4 (before the test meal) and day 5 or 6 (before the PET scan) of both study periods. On drug, plasma concentrations were lowest for sibutramine (mean $\pm \mathrm{SD}$ of both days: $2.03 \pm 1.19 \mathrm{ng} / \mathrm{ml}$ ), intermediate for M1 $(4.12 \pm 1.23 \mathrm{ng} / \mathrm{ml})$ and highest for M2 $(8.13 \pm 1.77 \mathrm{ng} / \mathrm{ml})$. On placebo, concentrations were non-detectable in all cases, as expected.
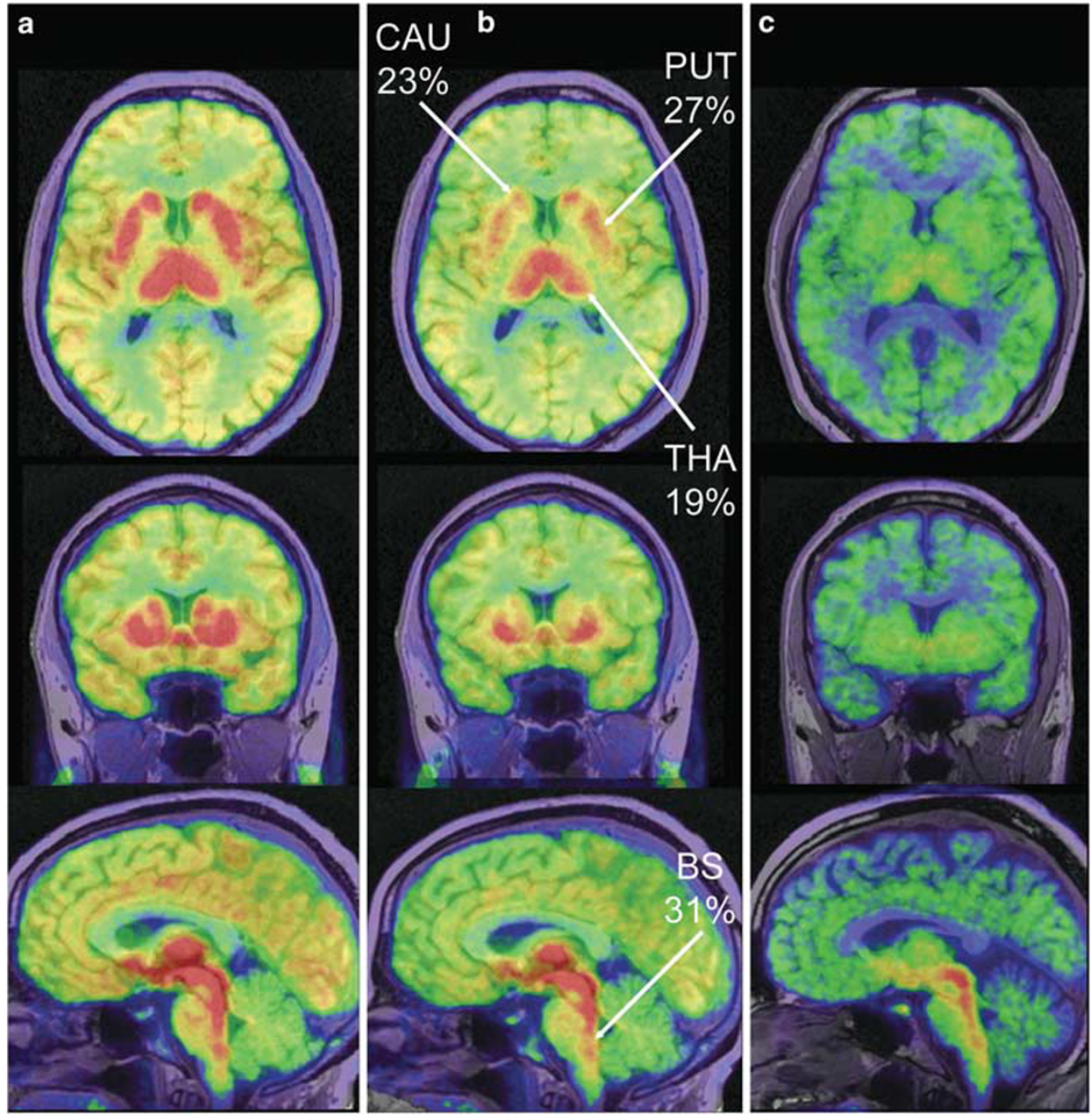

Figure I Summed I C-DASB PET data from 40-90 min post-injection, smoothed with $2.5 \mathrm{~mm}$ Gaussian filter for display purposes, normalized to injected dose (a-c: 604, 629 and $507 \mathrm{MBq}$, respectively), displayed at same intensity. (a and b) Placebo (a) and sibutramine (b) conditions in a representative 45 years old subject, illustrating the modest attenuation of signal in the caudate (CAU), putamen (PUT), thalamus (THA), and brainstem (BS) by sibutramine representing calculated regional occupancy values of 23, 27, 19, and 31\%, respectively, in this individual. (c) Placebo condition in a separate 49-year-old subject (labeled '5' in Figure 3), illustrating the extremely low baseline regional binding (of undetermined cause) in the CAU, PUT, and THA in this individual (see Relationship between SERT occupancy and food intake (in Sibutramine Pharmacodynamics) and Discussion sections for details). 
Concentrations of the three molecules achieved on the two test days did not significantly differ (RM ANOVA, concentration*test day: $p=0.71$ ) and were highly significantly correlated (sibutramine: $r^{2}=0.71, p<0.001 ; \mathrm{M} 1$ : $\left.r^{2}=0.83, p<0.0001 ; \mathrm{M} 2: r^{2}=0.65, p=0.002\right)$. These results confirm that sibutramine had reached steady state by day 4 and that SERT occupancy and PD data were acquired under comparable PK conditions.

Relationship between plasma concentrations and SERT occupancy. Blood samples for plasma concentrations of sibutramine, M1 and M2 were collected $12.9 \pm 3.2 \mathrm{~min}$ before radiotracer injection. To elucidate the relative contributions of the three molecules to overall SERT occupancy, plasma concentrations were regressed against measured SERT occupancy (mean of CAU, PUT, THA, and BS occupancy values) for each subject to look for a positive correlation (one-tailed) between increasing plasma concentration and SERT occupancy (see Figure 2). There was no significant relationship between SERT occupancy and plasma concentration of either sibutramine or M1 (Panels $a$ and $b)$. In contrast, a positive correlation $\left(r^{2}=0.167\right)$ was found between M2 and SERT occupancy (Panel c). Nevertheless, this reached only trend significance $(p=0.09)$, due largely to a subject (labeled ' 4 ' in Panel $c$ ) with low SERT occupancy (in fact the lowest of the group) despite relatively high M2 plasma concentration. Formal outlier analysis (standardized residual $>2$ ) of the data identified subject ' 4 ' as the only outlier, having a regression standardized residual of -2.33 . Removing this subject from the regression resulted in a much more robust correlation between M2 concentration and SERT occupancy, reaching high statistical significance $\left(r^{2}=0.59, p=0.003\right)$. Other than his outlier status, quality control of his PET data provided no scientific grounds for excluding this subject's data. We therefore present both results, with the full data set as the primary result.

\section{Sibutramine Pharmacodynamics}

Appetite suppression. For the whole group, mean food intake during the test meal was modestly but significantly reduced on sibutramine compared with placebo $(-12.2 \pm 14.7 \%$; paired $t$-test, $p=0.010)$. However, there was considerable variability in the magnitude of appetite change due to sibutramine across subjects ( +10.1 to $-38.5 \%)$. Based on response criteria ( $\geqslant 14.2 \%$ reduction; Figure 3, line A), six subjects were rated as responders to sibutramine (Figure 3, diamonds) and six as non-responders (Figure 3, circles).

Relationship between SERT occupancy and food intake. There was a positive relationship between SERT occupancy (mean of CAU, PUT, THA, and BS occupancy values) and appetite suppression (Figure 3, regression line B). Subjects with the lowest SERT occupancy had no appetite suppression, whereas those with the highest occupancy tended to have the greatest appetite suppression. However, this was not statistically significant $\left(r^{2}=0.077 ; p=0.19\right.$, one-tailed), largely because of three subjects (Figure 3, labeled ' 5 ', ' 6 ', and ' 7 ') with relatively high occupancy (36-39\%) but no appetite suppression. When responders and non-responders were compared, no response was seen at less than approximately $25 \%$ occupancy, all responders had occupancy in the mid to upper range, and the highest occupancy

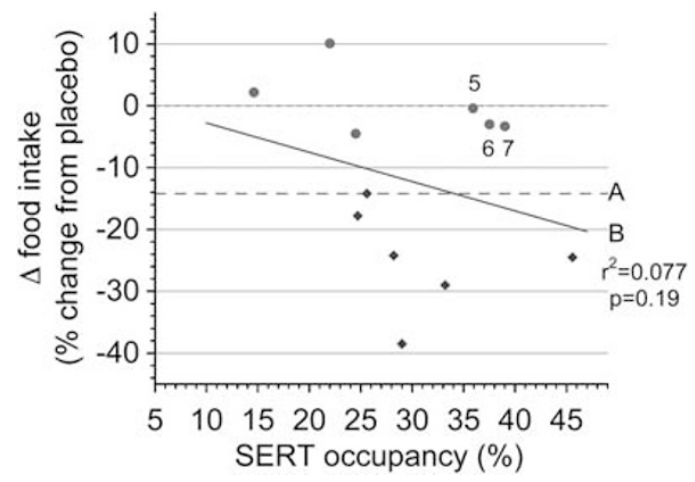

Figure 3 Serotonin reuptake transporter (SERT) occupancy by sibutramine regressed against change in food intake due to sibutramine for all subjects $(n=12)$. $Y$ axis: the zero line represents no change in appetite, and increasingly negative values represent increasing appetite suppression. Correlation and significance (one-tailed) values are indicated. Line A: test-retest variability (\% coefficient of variation) of the test meal model (14.2\%). Subjects whose magnitude of appetite suppression was $\geqslant 14.2 \%$ (diamonds; $n=6$ ) were designated 'responders'; subjects with appetite suppression $<14.2 \%$ were designated 'non-responders' (circles; $n=6$ ). The single occupancy value used for each subject is the mean of caudate, putamen, thalamus, and brain-stem occupancy values.
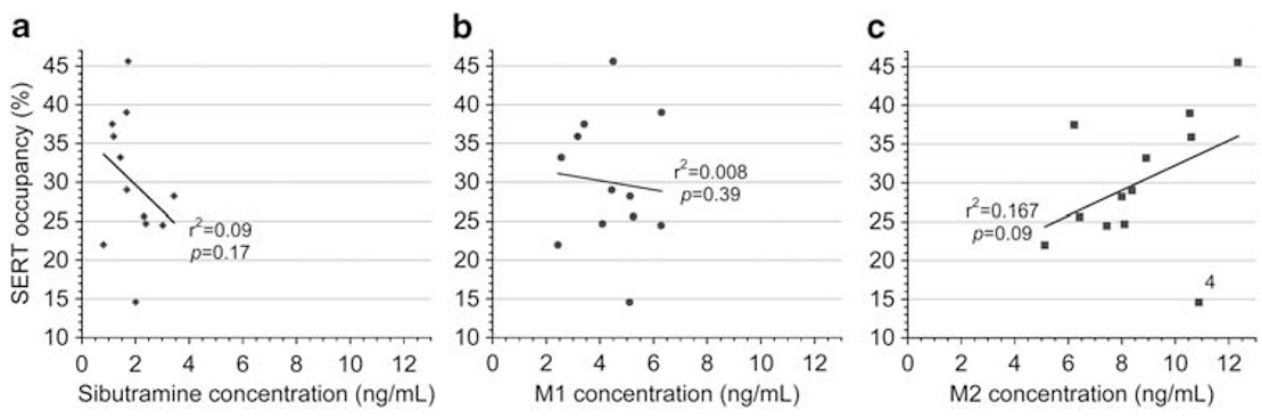

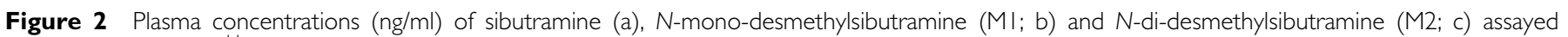

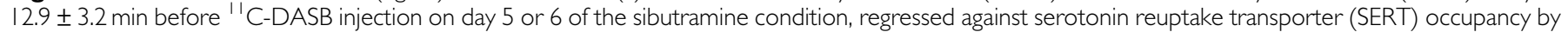

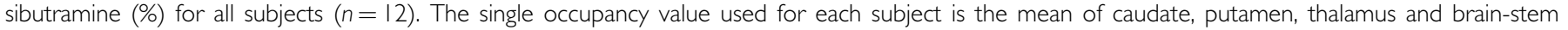
occupancy values. Correlations and significance (one-tailed) values are indicated. 
(45.6\%) was associated with a responder. In contrast, nonresponders had occupancy across a wide range (14.6$39.0 \%)$, and the lowest occupancy $(14.6 \%)$ was associated with a non-responder.

Subjects ' 5 ', ' 6 ', and ' 7 ' stand out as having no appetite suppression despite SERT occupancy in the upper range. Of these, subject ' 5 ' achieved relatively high plasma drug concentrations, but was found to have unusually low (less than two SD below the mean) values for baseline $\mathrm{BP}_{\mathrm{ND}}$ in THA (0.47), PUT (0.38), and CAU (0.19) and low (though normal) $\mathrm{BP}_{\mathrm{ND}}$ in $\mathrm{BS}(0.45)$ (see Figure 1, column C). Despite this, quality control of his radiochemistry and PET data, and his satisfaction of inclusion and exclusion criteria, gave no scientific grounds to exclude this subject's data. For subjects ' 6 ' and '7', no clearly unusual features were found for their $\mathrm{BP}_{\mathrm{ND}}$ or plasma concentrations of sibutramine, $\mathrm{M} 1$ and M2 that would explain the lack of appetite suppression.

\section{DISCUSSION}

In this study, we have measured, for the first time to the best of our knowledge, brain SERT occupancy by sibutramine in human subjects. The principal finding is that at the highest recommended dose (15 mg daily) SERT occupancy is of modest magnitude (mean $30.0 \pm 10.2 \%$ ), and varies widely across subjects (14.6-45.6\%). We found a positive correlation between SERT occupancy and plasma concentration of the M2 metabolite ( $N$-di-desmethylsibutramine), but not with parent sibutramine or M1 ( $N$-monodesmethylsibutramine) concentrations, suggesting that brain SERT occupancy may be predominantly mediated by $\mathrm{M} 2$ in humans taking sibutramine. Finally, we found a positive (but not statistically significant) relationship between SERT occupancy and the magnitude of appetite suppression, with no significant appetite suppression seen below approximately 25\% SERT occupancy and the greatest appetite suppression generally seen in those subjects with highest SERT occupancy. However, SERT occupancy poorly predicted appetite suppression, and several subjects had no appetite suppression despite SERT occupancy in the upper range. Taken together, these suggest that SERT occupancy may be necessary but is not by itself sufficient for appetite suppression in humans, supporting preclinical findings that appetite suppression by sibutramine requires co-inhibition of SERT and NET (Jackson et al, 1997b).

The limited pre-existing literature in animals and humans suggested that central SERT occupancy associated with $15 \mathrm{mg}$ of sibutramine daily may be very low or even indistinguishable from placebo (Luscombe et al, 1990; Thomas et al, 2009). We have demonstrated that it can be measured accurately by ${ }^{11} \mathrm{C}-\mathrm{DASB}$ PET and, although of modest magnitude (mean $30 \%$ ), is clearly not negligible in humans. This magnitude is in contrast to SSRIs and other SERT-binding antidepressant drugs, which typically achieve $80 \%$ regional SERT occupancy, particularly in striatum, at clinically effective doses (Meyer et al, 2004; Talbot and Laruelle, 2002). Sibutramine was initially developed in the 1980s as a potential antidepressant (Buckett et al, 1988), and indeed the $K_{\mathrm{i}}$ values of M1 and M2 against ${ }^{3} \mathrm{H}-5-\mathrm{HT}$ uptake in vitro are comparable to the antidepressants fluoxetine, amitriptyline, and imipramine (Cheetham et al, 1993; Heal et al, 1998). However, it was found in Phase II clinical trials to be ineffective as an antidepressant at the doses tested (Kelly et al, 1995 (abstract) quoted in Heal et al, 1998) and to have an unacceptable tolerability and safety profile at higher doses. The relatively poor predictive value of small animal data, and our finding of only modest SERT occupancy at the maximum tolerable dose, provides support for using in vivo occupancy with PET early in the drug development process.

Nevertheless, a mean sibutramine occupancy of $30 \%$ clearly raises the potential in clinical practice for adverse drug interactions and reinforces current clinical safety warnings. The manufacturers contraindicate the concurrent use of sibutramine with monoamine reuptake inhibitors (MAOIs), and advise that it should not be given with other serotonergic drugs (including SSRIs, other antidepressants, tryptophan, ergolines, tryptans, opioids, and lithium) because of the risk of the serious serotonin syndrome (Baxter, 2007; BNF, 2009).

The rank order of the sibutramine $(2.03 \pm 1.19), \mathrm{M} 1$ $(4.12 \pm 1.23)$ and M2 $(8.13 \pm 1.77 \mathrm{ng} / \mathrm{ml})$ plasma concentrations is consistent with previous findings that M2 plasma concentration is higher and elimination slower than M1 in humans (Hind et al, 1999). We found no positive relationship between central SERT occupancy and plasma concentration of parent sibutramine. This would be consistent with its short $t_{\max }(\sim 1 \mathrm{~h})$, rapid plasma washout $\left(t_{\frac{1}{2}} \sim 1 \mathrm{~h}\right)$, and the very low in vitro potency for 5-HT reuptake inhibition ( 2000-3000 nM) found in rat tissue (Cheetham et al, 1993; Heal et al, 1998). The most likely explanation for the negative nature of the relationship (see Figure 2, left hand panel) may be that this is a reflection of the positive relationship between SERT occupancy and plasma concentration of M2 (Figure 2, right hand panel). That is, higher levels of M2 because of more rapid or extensive hepatic metabolism of sibutramine must be reflected in lower residual levels of the parent. The lack of a positive relationship between plasma M1 concentration and central SERT occupancy (Figure 2, central panel) was somewhat surprising given evidence that racemic M1 and M2 have broadly similar in vitro potency for 5-HT reuptake inhibition (M1: $\sim 18 \mathrm{nM}$; M2: $\sim 25 \mathrm{nM}$ ) in rat tissue (Cheetham et al, 1993; Heal et al, 1998). Possible explanations include species differences between rats and humans, lower M1 concentrations in plasma (approximately $50 \%$ of the M2 concentration) and brain, and the fact that M2 is generated solely by demethylation of M1 (as M1 is generated solely by demethylation of parent sibutramine, Hakala et al, 2009; Link et al, 2006) so that increasing plasma M2 must necessarily be accompanied by decreasing plasma M1 and subsequent faster washout of M1 than M2 from CNS.

The finding of a relationship between SERT occupancy and M2 rather than M1 may have relevance to future drug development. Glick et al (2000) have suggested that the metabolites might be safer anti-obesity agents than parent sibutramine and have shown that the $(R)$-enantiomers of the metabolites have 1-2 orders of magnitude more potent 5 -HT reuptake inhibition than the (S)-enantiomers. Our data and the enantioselective findings of Glick et al (2000) suggest that M2, particularly (R)-M2, may be a promising potential anti-obesity drug candidate. Nevertheless, the 
potential contribution of M1 to appetite suppressant efficacy via mechanisms not measured in this study (eg, NET occupancy) cannot be excluded.

Our data would support the conclusion that lack of appetite suppression in some subjects may result from multiple mechanisms. For some, inadequate SERT occupancy would appear to be due to low plasma concentration of M2 (Figure 2, right hand panel). The cytochrome P450 isoenzyme CYP2B6 has been identified as the major enzyme responsible for the sequential demethylations of sibutramine to $\mathrm{M} 1$ and $\mathrm{M} 1$ to $\mathrm{M} 2$ in vitro, with minor contributions from 2C19, 3A4, and 3A5 (Bae et al, 2008). CYP2B6 polymorphism is common in white populations (see original references in Bae et al, 2008), suggesting that genetic variability, particularly in CYP2B6 activity, may have a major role in the interindividual variability in bioavailability of $\mathrm{M} 1$ and M2 seen in vivo. For others, central SERT occupancy is low despite relatively high plasma M2 levels (Figure 2, right hand panel, label ' 4 '). Possible mechanisms include the well-recognized potential for differing ratios of parent drug and metabolites between blood and CNS, variability in blood-brain barrier mechanisms, and variability in a plasma-free (unbound) drug. M1 and M2 are both highly protein bound in plasma (94\%) so that small between-subject differences in protein binding may lead to large differences in the concentration of free drug available to enter the CNS. For still other subjects, non-response may be due to biological heterogeneity in SERT mechanisms. For example, one subject with no appetite suppression on sibutramine (Figure 3, label ' 5 ') was found to have extremely low SERT availability in THA, PUT, and CAU (Figure 1, column C), raising the possibility of a biological explanation for his non-response related to abnormal SERT availability or function. Firm evidence now exists for genetically and environmentally mediated variability in SERT density and activity between subjects. For example, a common triallelic polymorphism at the serotonin transporter-linked polymorphic region (5-HTTLPR) on chromosome $17 \mathrm{q} 12$ impacts on SERT mRNA transcription and confers variability in SERT expression that is measurable by ${ }^{11} \mathrm{C}$-DASB PET (Praschak-Rieder et al, 2007; Reimold et al, 2007). Environmental factors include seasonal variation in SERT availability (Praschak-Rieder et al, 2008) and evidence that childhood adversity may be associated with a persisting reduction of SERT availability in adulthood (Miller et al, 2009). A recent PET study has found low SERT to be associated with poor treatment response to other drugs (antidepressants) acting largely via monoamine reuptake inhibition (Miller et al, 2008). Interestingly, two (relatively small) pharmacogenetic studies have found that 5-HTTLPR polymorphisms may also be associated with differential clinical response to sibutramine (Grudell et al, 2008; Vazquez Roque et al, 2007). In our study, subjects were not genotyped and we did not screen for a past history of environmental adversity such as childhood abuse. Nor can we completely exclude the possibility that this subject might have deliberately misled us about recent misuse of, for example, MDMA, although his urine toxicology at screening and study participation was negative. Nevertheless, this subject's non-response, his very low baseline SERT availability, the existence of recognized biological mechanisms giving rise to measurable variability in SERT expression and function, and pharmacogenetic data linking 5-HTTLPR polymorphisms with response to sibutramine, suggest that future studies should further investigate hypothesized genetic and environmental SERT-related mechanisms for non-response to 5-HT reuptake inhibitor drugs including sibutramine and antidepressants. An alternative explanation for the low regional $\mathrm{BP}_{\mathrm{ND}}$ values measured in subject ' 5 ' may be abnormally poor ${ }^{11} \mathrm{C}$-DASB binding in this subject giving rise to apparent rather than real reductions in SERT availability. Although this could be caused (at least theoretically) by conformational abnormalities in the SERT protein, we feel that this is a less likely explanation and has not (to the best of our knowledge) been previously reported for this radiotracer. Lastly, for other subjects, lack of appetite suppression was seen despite normal baseline SERT availability and relatively high levels of SERT occupancy by sibutramine (Figure 3, labels ' 6 ' and ' 7 '), suggesting that non-response in these subjects may be related to non-SERT mechanisms such as NET inhibition, or psychological and environmental factors.

Our study has a number of strengths, in particular the double-blind, placebo-controlled, balanced-order, withinsubject crossover design. The cohort size $(n=12)$ is relatively large for a drug occupancy study, and the excellent imaging qualities and test-retest reliability of ${ }^{11} \mathrm{C}$-DASB in the chosen ROIs maximize the study's potential to measure between-condition differences in SERT binding (Frankle et al, 2004, 2006; Talbot et al, 2005). We have made considerable effort to exclude confounding SERT-related factors in our choice of exclusion criteria and screening. There were no systematic differences in radiochemistry parameters or post-dose delay before PET scanning between the sibutramine and placebo conditions, and we have demonstrated that sibutramine had reached steady state by day 4 ensuring that PD and PET data were acquired under similar PK conditions. It is known from the literature that the pharmacokinetics of M1 and M2 following a single dose of $15 \mathrm{mg}$ sibutramine are not significantly different between young (mean age 24.0 years) and elderly (mean age 70.3 years) healthy subjects (Hind et al, 1999), suggesting that the age range of our subjects $(30-50)$ is not a large contributor to the variability in the data.

We examined the data for an effect of condition order (sibutramine-placebo $v s$ placebo-sibutramine) on the regional $\mathrm{BP}_{\mathrm{ND}}$ values in the placebo condition to test for possible evidence of residual CNS sibutramine suppressing the placebo $\mathrm{BP}_{\mathrm{ND}}$ of subjects scanned in the sibutramineplacebo order. We did in fact find a (just) significant effect of order $(p=0.048)$. However, placebo $\mathrm{BP}_{\mathrm{ND}}$ was higher (by a mean of $15 \%$ ) in the subjects scanned in the order 'sibutramine-placebo' when compared to those scanned in the order 'placebo-sibutramine'. This is the opposite of what would be expected if residual sibutramine from the first scan was still occupying SERT during the second (placebo) scan. Our data suggest that the 13-15 day washout between PET scans is adequate and we found no evidence for residual occupancy by sibutramine at this time point. However, the higher placebo regional $\mathrm{BP}_{\mathrm{ND}}$ values in those subjects who received sibutramine 2 weeks previously is unexpected. The data set is very small, the observation 
requires replication in a larger data set, and any conjecture of mechanism goes beyond the data. However, one possible explanation may be that the short-term sibutramine occupancy has led to an increase in the SERT $B_{\text {avail }}$ (density of transporters available to bind radioligand in vivo, Innis et al, 2007) or affinity for ${ }^{11} \mathrm{C}$-DASB. We report this as an interesting observation warranting future investigation.

We acknowledge that the study has several limitations. First, in clinical practice the prescription of sibutramine is limited to obese patients, for up to 1 year, and approximately $80 \%$ of patients in England are female (Perrio et al, 2007). This may therefore limit the extrapolation of our results (healthy males of normal BMI) to sibutramine's target population. Available published pharmacokinetic data are relatively reassuring, as $C_{\max }, t_{\max }$ and elimination $t_{\frac{1}{2}}$ for M1 and M2 do not appear to be significantly different whether measured in obese patients (BMI $30-42 \mathrm{~kg} / \mathrm{m}^{2}$ ) or healthy volunteers, and steady state has been shown to be maintained between 12 and 24 weeks (McNeely and Goa, 1998). Nevertheless, we were unable to find information on potential gender effects, the appetite suppression seen after 4 days in healthy males has limitations as a model for the chronic use of sibutramine (Halford et al, 2007), and the possibility remains that SERT occupancy levels could be affected by demographic differences between our study cohort and the drug's target population. Second, although the cohort size is clearly adequate to measure betweenconditions differences in SERT occupancy (the primary aim of the study), the study has low power for the correlations between occupancy and PK and PD measures. This risk was recognized a priori, and the correlations were considered to be exploratory secondary aims. We felt that, in the absence of pilot data, a cohort size of 12 was a reasonable balance between fulfilling the aims of the study and the risks of exposing an even larger cohort to radiation without acquiring useful data in return. Future studies can use our data to inform accurate power calculations. Third, it was not possible in practice to start all $24 \mathrm{PET}$ scans between 240 and 270 min post-dose. For two subjects, their first PET scan was unavoidably delayed beyond $270 \mathrm{~min}$ for technical reasons. We succeeded in delaying their second PET scan by the same duration $( \pm 30 \mathrm{~min})$ so that ${ }^{11} \mathrm{C}-\mathrm{DASB} \mathrm{BP}_{\mathrm{ND}}$ in the two conditions would be measured under comparable $\mathrm{PK}$ conditions. However, this necessarily introduces some increased variability into the correlation between SERT occupancy and the PD measure across the group, as food intake was still measured at $240 \mathrm{~min}$ post-dose in both conditions for these 2 subjects. For 2 other subjects, their first scan was performed shortly after $240 \mathrm{~min}$ but their second scan was unavoidably delayed slightly beyond $270 \mathrm{~min}$. However, given the long $t_{\frac{1}{2}}$ of M1 and M2 (14-19 h; that is, a decline in concentration after $t_{\max }$ of $3-4 \%$ per hour, at most), we would contend that the additional delays in these four subjects are unlikely to have materially affected the results.

In summary, our data support the conclusion that 5-HT reuptake inhibition may be necessary but is not sufficient for the appetite suppressant effect of clinically recommended doses of sibutramine in humans, and provide a basis for the development of future PET studies to measure NET occupancy by sibutramine at the same dose.

\section{ACKNOWLEDGEMENTS}

We gratefully acknowledge the technical and study management contributions of the following personnel: Campbell McCullough, Elizabeth Barnett, Steve Nolan, Jon Lowe, Katrina Copeland, Carrie-Anne Mellor, Mike Green, Mike Fairclough, Lynne Macrae, Kathryn Marley, Lloyd Gregory and Barry Whitnall. We thank Rainer Hinz for helpful discussions and Professor Alan Jackson for providing MRI clinical reports. MRI scans were acquired using the facilities of the Wellcome Trust Clinical Research Facility, Manchester. Selected data from this study were presented, in abstract form, at the Society of Nuclear Medicine 56th Annual Meeting, Toronto, Canada, 13-17 June 2009; and the IXth International Conference on Quantification of Brain Function with PET, Chicago, USA, 30 June to 1 July 2009.

\section{DISCLOSURE}

All authors confirm they have no conflict of interest in this study. In particular, none of the authors has any association with Abbott Laboratories, the manufacturer of sibutramine. PST has received small honoraria from the British Association for Psychopharmacology for teaching in each of the last 3 years; AM has received small honoraria from the University of Wolverhampton as an external examiner in each of the last 3 years; CPC is a salaried employee of Icon PLC, the sponsors of the study, and holds stock options in ICON PLC; at the time of study conduct, SB and AWP were salaried employees of Icon PLC and held stock options in ICON PLC.

\section{REFERENCES}

Arterburn DE, Crane PK, Veenstra DL (2004). The efficacy and safety of sibutramine for weight loss: a systematic review. Arch Intern Med 164: 994-1003.

Babalola KO, Cootes TF, Twining CJ, Petrovic V, Taylor CJ (2008). $3 \mathrm{D}$ brain segmentation using active appearance models and local regressors. Proceedings of the 11th International Conference on Medical Image Computing and Computer Assisted Intervention (MICCAI), Lecture Notes in Computer Science 5241: 401-408.

Bae SK, Cao S, Seo K-A, Kim H, Kim M-J, Shon J-H et al (2008). Cytochrome P450 2B6 catalyzes the formation of pharmacologically active sibutramine (N-\{1-[1-(4-chlorophenyl)cyclobutyl]3 -methylbutyl $\}-N, N$-dimethylamine) metabolites in human liver microsomes. Drug Metab Dispos 36: 1679-1688.

Baxter K (ed). (2007). Stockley's Drug Interactions, 8th Revised edn. Pharmaceutical Press: London, UK.

BNF (2009). Joint Formulary Committee. British National Formulary, 57th edn. British Medical Association and Royal Pharmaceutical Society of Great Britain: London.

Buckett WR, Thomas PC, Luscombe GP (1988). The pharmacology of sibutramine hydrochloride (BTS 54 524), a new antidepressant which induces rapid noradrenergic down-regulation. Prog Neuropsychopharmacol Biol Psychiatry 12: 575-584.

Byars LG, Sibomana M, Burbar Z, Jones J, Panin V, Barker WC; et al (2005). Variance reduction on randoms from coincidence histograms for the HRRT. IEEE Nuclear Science Symposium Conference Record 5: 2622-2626.

Cheetham SC, Viggers JA, Slater NA, Heal DJ, Buckett WR (1993). $[3 \mathrm{H}]$ paroxetine binding in rat frontal cortex strongly correlates with $[3 \mathrm{H}] 5-\mathrm{HT}$ uptake: effect of administration of various antidepressant treatments. Neuropharmacology 32: 737-743. 
de Jong HWAM, van Velden FHP, Kloet RW, Buijs FL, Boellaard R, Lammertsma AA (2007). Performance evaluation of the ECAT HRRT: an LSO-LYSO double layer high resolution, high sensitivity scanner. Phys Med Biol 52: 1505-1526.

Frankle WG, Huang Y, Hwang D-R, Talbot PS, Slifstein M, Van Heertum $\mathrm{R}$ et al (2004). Comparative evaluation of serotonin transporter radioligands $11 \mathrm{C}-\mathrm{DASB}$ and $11 \mathrm{C}-\mathrm{McN} 5652$ in healthy humans. J Nucl Med 45: 682-694.

Frankle WG, Slifstein M, Gunn RN, Huang Y, Hwang D-R, Darr EA et al (2006). Estimation of serotonin transporter parameters with $11 \mathrm{C}-\mathrm{DASB}$ in healthy humans: reproducibility and comparison of methods. J Nucl Med 47: 815-826.

Garratt CJ, Hind ID, Haddock RE (1995). Single/repeat dose kinetics of sibutramine metabolites in obese subjects. J Clin Pharmacol 35: 928 (abstract).

Glick SD, Haskew RE, Maisonneuve IM, Carlson JN, Jerussi TP (2000). Enantioselective behavioral effects of sibutramine metabolites. Eur J Pharmacol 397: 93-102.

Grignaschi G, Fanelli E, Scagnol I, Samanin R (1999). Studies on the role of serotonin receptor subtypes in the effect of sibutramine in various feeding paradigms in rats. Br J Pharmacol 127: $1190-1194$.

Grudell ABM, Sweetser S, Camilleri M, Eckert DJ, Vazquez-Roque MI, Carlson PJ et al (2008). A controlled pharmacogenetic trial of sibutramine on weight loss and body composition in obese or overweight adults. Gastroenterology 135: 1142-1154.

Hainer V, Kabrnova K, Aldhoon B, Kunesova M, Wagenknecht M (2006). Serotonin and norepinephrine reuptake inhibition and eating behavior. Ann NY Acad Sci 1083: 252-269.

Hakala KS, Link M, Szotakova B, Skalova L, Kostiainen R, Ketola RA (2009). Characterization of metabolites of sibutramine in primary cultures of rat hepatocytes by liquid chromatography-ion trap mass spectrometry. Anal Bioanal Chem 393: 1327-1336.

Halford JCG, Harrold JA, Boyland EJ, Lawton CL, Blundell JE (2007). Serotonergic drugs: effects on appetite expression and use for the treatment of obesity. Drugs 67: 27-55.

Heal DJ, Cheetham SC, Prow MR, Martin KF, Buckett WR (1998). A comparison of the effects on central 5-HT function of sibutramine hydrochloride and other weight-modifying agents. Br J Pharmacol 125: 301-308.

Hill AJ, Rogers PJ, Blundell JE (1995). Techniques for the experimental measurement of human eating behaviour and food intake: a practical guide. Int J Obes Relat Metab Disord 19: 361-375.

Hind ID, Mangham JE, Ghani SP, Haddock RE, Garratt CJ, Jones RW (1999). Sibutramine pharmacokinetics in young and elderly healthy subjects. Eur J Clin Pharmacol 54: 847-849.

Huang Y, Narendran R, Bae S-A, Erritzoe D, Guo N, Zhu Z et al (2004). A PET imaging agent with fast kinetics: synthesis and in vivo evaluation of the serotonin transporter ligand [11C]2[2-dimethylaminomethylphenylthio)]-5-fluorophenylamine ([11C] AFA). Nucl Med Biol 31: 727-738.

Innis RB, Cunningham VJ, Delforge J, Fujita M, Gjedde A, Gunn $\mathrm{RN}$ et al (2007). Consensus nomenclature for in vivo imaging of reversibly binding radioligands. J Cereb Blood Flow Metab 27: $1533-1539$.

Jackson HC, Bearham MC, Hutchins LJ, Mazurkiewicz SE, Needham AM, Heal DJ (1997a). Investigation of the mechanisms underlying the hypophagic effects of the 5-HT and noradrenaline reuptake inhibitor, sibutramine, in the rat. $\mathrm{Br}$ J Pharmacol 121: 1613-1618.

Jackson HC, Needham AM, Hutchins LJ, Mazurkiewicz SE, Heal DJ (1997b). Comparison of the effects of sibutramine and other monoamine reuptake inhibitors on food intake in the rat. $\mathrm{Br}$ J Pharmacol 121: 1758-1762.

Kelly F, Jones SP, Lee JK (1995). Sibutramine: weight loss in depressed patients. Int J Obes Relat Metab Disord 19: p397.
King DJ, Devaney N (1988). Clinical pharmacology of sibutramine hydrochloride (BTS 54524), a new antidepressant, in healthy volunteers. Br J Clin Pharmacol 26: 607-611.

Kissileff HR, Klingsberg G, Van Itallie TB (1980). Universal eating monitor for continuous recording of solid or liquid consumption in man. Am J Physiol 238: R14-R22.

Larsen P, Ulin J, Dahlstrom K, Jensen M (1997). Synthesis of [11C]iodomethane by iodination of [11C]methane. Appl Radiat Isot 48: 153.

Li Z, Maglione M, Tu W, Mojica W, Arterburn D, Shugarman LR et al (2005). Meta-analysis: pharmacologic treatment of obesity. Ann Intern Med 142: 532-546.

Link M, Hakala KS, Wsol V, Kostiainen R, Ketola RA (2006). Metabolite profile of sibutramine in human urine: a liquid chromatography-electrospray ionization mass spectrometric study. J Mass Spectrom 41: 1171-1178.

Liu YL, Heal DJ, Stock MJ (2002). Mechanism of the thermogenic effect of Metabolite 2 (BTS 54505), a major pharmacologically active metabolite of the novel anti-obesity drug, sibutramine. Int $J$ Obes Relat Metab Disord 26: 1245-1253.

Liu YL, Kashani SMZ, Heal DJ, Stock MJ (1996). Effect of sibutramine on tissue glucose utilisation in the rat. $\mathrm{Br} J$ Pharmacol 117: 324P.

Logan J, Fowler JS, Volkow ND, Wang GJ, Ding YS, Alexoff DL (1996). Distribution volume ratios without blood sampling from graphical analysis of PET data. J Cereb Blood Flow Metab 16: 834-840.

Luscombe GP, Hopcroft RH, Thomas PC, Buckett WR (1989). The contribution of metabolites to the rapid and potent downregulation of rat cortical beta-adrenoceptors by the putative antidepressant sibutramine hydrochloride. Neuropharmacology 28: $129-134$.

Luscombe GP, Slater NA, Lyons MB, Wynne RD, Scheinbaum ML, Buckett WR (1990). Effect on radiolabelled-monoamine uptake in vitro of plasma taken from healthy volunteers administered the antidepressant sibutramine $\mathrm{HCl}$. Psychopharmacology (Berl) 100: 345-349.

McNeely W, Goa KL (1998). Sibutramine. A review of its contribution to the management of obesity. Drugs 56: 1093-1124.

Meyer JH (2007). Imaging the serotonin transporter during major depressive disorder and antidepressant treatment. J Psychiatry Neurosci 32: 86-102.

Meyer JH, Wilson AA, Sagrati S, Hussey D, Carella A, Potter WZ et al (2004). Serotonin transporter occupancy of five selective serotonin reuptake inhibitors at different doses: an [11C]DASB positron emission tomography study. Am J Psychiatry 161: 826-835.

Michel C, Schmand M, Liu X, Sibomana M, Vollmar S, Knoss C et al (2000). Reconstruction strategies for the HRRT. IEEE Nuclear Science Symposium Conference Record 2: 15/207-215/ 212.

Miller JM, Kinnally EL, Ogden RT, Oquendo MA, Mann JJ, Parsey RV (2009). Reported childhood abuse is associated with low serotonin transporter binding in vivo in major depressive disorder. Synapse 63: 565-573.

Miller JM, Oquendo MA, Ogden RT, Mann JJ, Parsey RV (2008). Serotonin transporter binding as a possible predictor of oneyear remission in major depressive disorder. J Psychiatr Res 42: $1137-1144$.

Padwal RS, Rucker D, Li SK, Curioni C, Lau DCW (2003). Longterm pharmacotherapy for obesity and overweight. Cochrane Database Syst Rev, Issue 4. Art. No.: CD004094. doi:10.1002/ 14651858.CD004094.pub2.

Perrio MJ, Wilton LV, Shakir SAW (2007). The safety profiles of orlistat and sibutramine: results of prescription-event monitoring studies in England. Obesity 15: 2712-2722.

Praschak-Rieder N, Kennedy J, Wilson AA, Hussey D, Boovariwala A, Willeit M et al (2007). Novel 5-HTTLPR allele associates with 
higher serotonin transporter binding in putamen: a [(11)C] DASB positron emission tomography study. Biol Psychiatry 62: 327-331.

Praschak-Rieder N, Willeit M, Wilson AA, Houle S, Meyer JH (2008). Seasonal variation in human brain serotonin transporter binding. Arch Gen Psychiatry 65: 1072-1078.

Reimold M, Smolka MN, Schumann G, Zimmer A, Wrase J, Mann $\mathrm{K}$ et al (2007). Midbrain serotonin transporter binding potential measured with [11C]DASB is affected by serotonin transporter genotype. J Neural Transm 114: 635-639.

Sibomana M, Byars L, Panin V, Lenox M, Kehren F, Rist J et al (2004). Simultaneous measurement of transmission and emission contamination using a collimated $137 \mathrm{Cs}$ point source for the HRRT. IEEE Nuclear Science Symposium Conference Record 4: 2647-2651.

Stunkard A, Messick S (1985). The Three-factor eating questionnaire to measure dietary restraint, disinhibition and hunger. J Psychosom Res 29: 71-83.

Talbot PS, Frankle WG, Hwang D-R, Huang Y, Suckow RF, Slifstein $\mathrm{M}$ et al (2005). Effects of reduced endogenous 5-HT on the in vivo binding of the serotonin transporter radioligand 11C-DASB in healthy humans. Synapse 55: 164-175.

Talbot PS, Laruelle M (2002). The role of in vivo molecular imaging with PET and SPECT in the elucidation of psychiatric drug action and new drug development. Eur Neuropsychopharmacol 12: $503-511$.

Thomas GH, Babbs AJ, Chatfield RE, Krulle TM, Widdowson PS, Provost D et al (2009). 5-HT(1A) activation counteracts cardiovascular but not hypophagic effects of sibutramine in rats. Obesity 17: 467-473.

van Velden FHP, Kloet RW, van Berckel BNM, Lammertsma AA, Boellaard R (2009). Accuracy of 3-dimensional reconstruction algorithms for the high-resolution research tomograph. $\mathrm{J} \mathrm{Nucl}$ Med 50: 72-80.

Vazquez Roque MI, Camilleri M, Clark MM, Tepoel DA, Jensen $\mathrm{MD}$, Graszer KM et al (2007). Alteration of gastric functions and candidate genes associated with weight reduction in response to sibutramine. Clin Gastroenterol Hepatol 5: 829-837.

Watson CC (2000). New, faster, image-based scatter correction for 3D PET. Nuclear Science, IEEE Transactions 47(Part 2): 1587-1594.

Weintraub M, Rubio A, Golik A, Byrne L, Scheinbaum ML (1991). Sibutramine in weight control: a dose-ranging, efficacy study. Clin Pharmacol Ther 50: 330-337.

Wilson AA, Ginovart N, Schmidt M, Meyer JH, Threlkeld PG, Houle S (2000). Novel radiotracers for imaging the serotonin transporter by positron emission tomography: synthesis, radiosynthesis, and in vitro and ex vivo evaluation of (11)C-labeled 2(phenylthio)araalkylamines. J Med Chem 43: 3103-3110. 